\title{
THORACOSCOPIC REPAIR OF A SPONTANEOUS PERFORATION OF THE ESOPHAGUS WITH THE ENDOSCOPIC SUTURING DEVICE
}

Yoshifumi Ikeda, MD, Masanori Niimi, MD, PhD, Yuzo Sasaki, MD, Tomoo Shatari, MD, Hiroshi Takami, MD, and

Susumu Kodaira, MD, Tokyo, Japan

Spontaneous perforation of the esophagus (Boerhaave's syndrome) is a life-threatening condition demanding early diagnosis and effective treatment to prevent fulminant mediastinitis and death. Primary closure and wide drainage of the mediastinum is generally accepted as the treatment of choice within the first 24 hours of spontaneous perforation of the esophagus. ${ }^{1,2}$ Although minimal access surgery with endoscopy has recently received much attention in various surgical procedures, only 1 case of thoracoscopic repair of transmural rupture of the esophagus has been reported. ${ }^{3}$ In this case, the repair was performed with standard free-needle suturing, but a leak occurred after the operation. We report the first successful case of thoracoscopic repair of a spontaneous ruputure of the esophagus with the use of the Endo Stitch loading unit (Auto Suture Company, Division of United States Surgical Corporation, Norwalk, Conn).

Clinical summary. A 39-year-old man was admitted 12 hours after forceful vomiting following alcohol consumption. He had progressive chest pain that was sharp and radiated to the back. He was unable to lie in the supine position. On examination, he was febrile $\left(39^{\circ} \mathrm{C}\right)$ and tachycardic (120 breaths/min). Blood pressure was $100 / 60 \mathrm{~mm} \mathrm{Hg}$. Chest radiograph revealed a left pleural effusion, mediastinal shift to the right, and mediastinal emphysema. A meglumine diatrizoate esophagogram confirmed left-sided rupture of the lower esophagus with shifting of the middle third of the esophagus to the right (Fig 1).

A double-lumen endobronchial tube was introduced for selective single-lung ventilation with the patient under general anesthesia. The man was placed in the right decubitus position. Five thoracoports were introduced with the collapsed left lung. One port was for a 10-mm end-viewing video camera, two other ports were for placement of endobronchial retractors, and the other two ports were for operating instruments. The pleural cavity was irrigated clean, and the inferior pulmonary ligament was divided to allow easy access to the

From the First Department of Surgery, Teikyo University School of Medicine, Tokyo, Japan.

Received for publication May 23, 2000; accepted for publication May 27, 2000.

Address for reprints: Yoshifumi Ikeda, First Department of Surgery, Teikyo University School of Medicine, 2-11-1 Kaga, Itabashi-ku,

Tokyo 173-8605, Japan (E-mail: yikeda@med.teikyo-u.ac.jp)

J Thorac Cardiovasc Surg 2001;121:178-9

Copyright () 2001 by The American Association for Thoracic Surgery

0022-5223/2001 \$35.00+ $0 \quad \mathbf{1 2 / 5 4 / 1 0 9 2 4 4}$

doi:10.1067/mtc.2001.109244
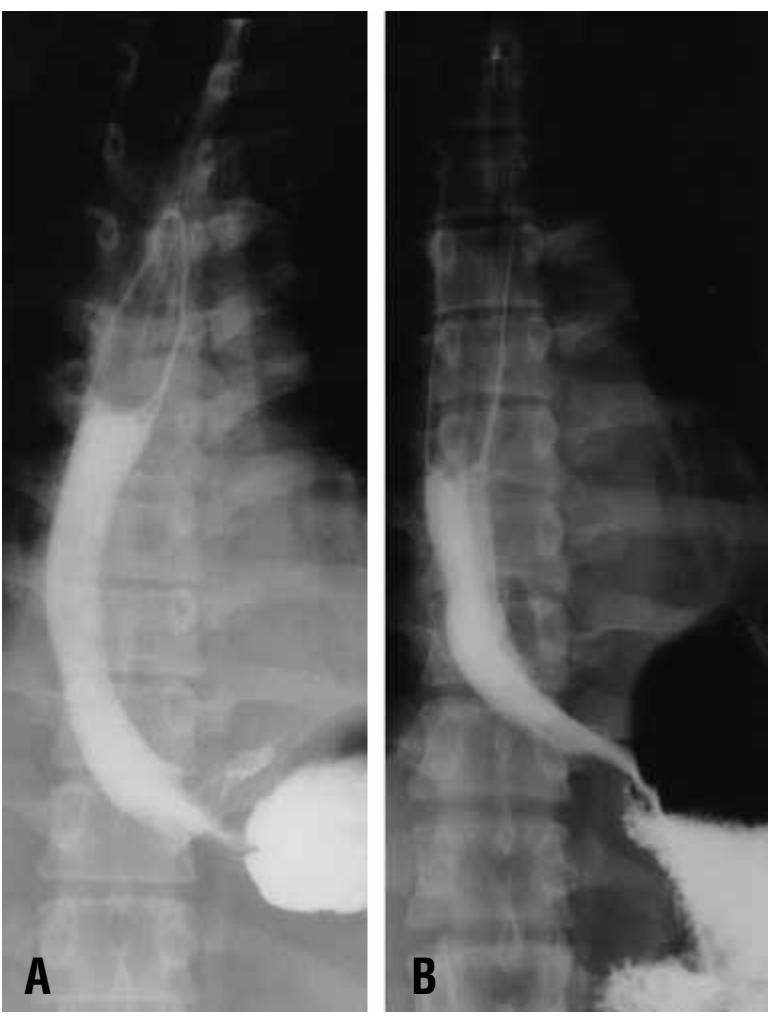

Fig 1. A meglumine diatrizoate esophagogram showing leftsided rupture of the lower esophagus and a shifting of the middle third of the esophagus before the operation (A). Seven days after the operation, there was no extravasation, and shifting of the middle third of the esophagus has improved (B).

lower esophagus. After gentle mobilization of the posterior mediastinum, we reached the site of the ruptured esophagus. An approximately $3-\mathrm{cm}$ longitudinal perforation of the left side of the esophagus was clealy visible. The ruptured site was repaired with the Endo Stitch loading unit. A needle with a Polysorb 3-0 suture (Auto Suture Company) was passed through the posterior wall first and then passed through the anterior wall. The suture was inserted in an interrupted fashion with intracorporeal maneuvers (Fig 2). This procedure was performed three times. The pleural cavity was re-irrigated and two chest drains were placed in the posterior thoracic cavity close to the repaired site under thoracoscopic direct vision. The operative time was approximately 1 hour. The patient recovered without any complications. A meglumine 

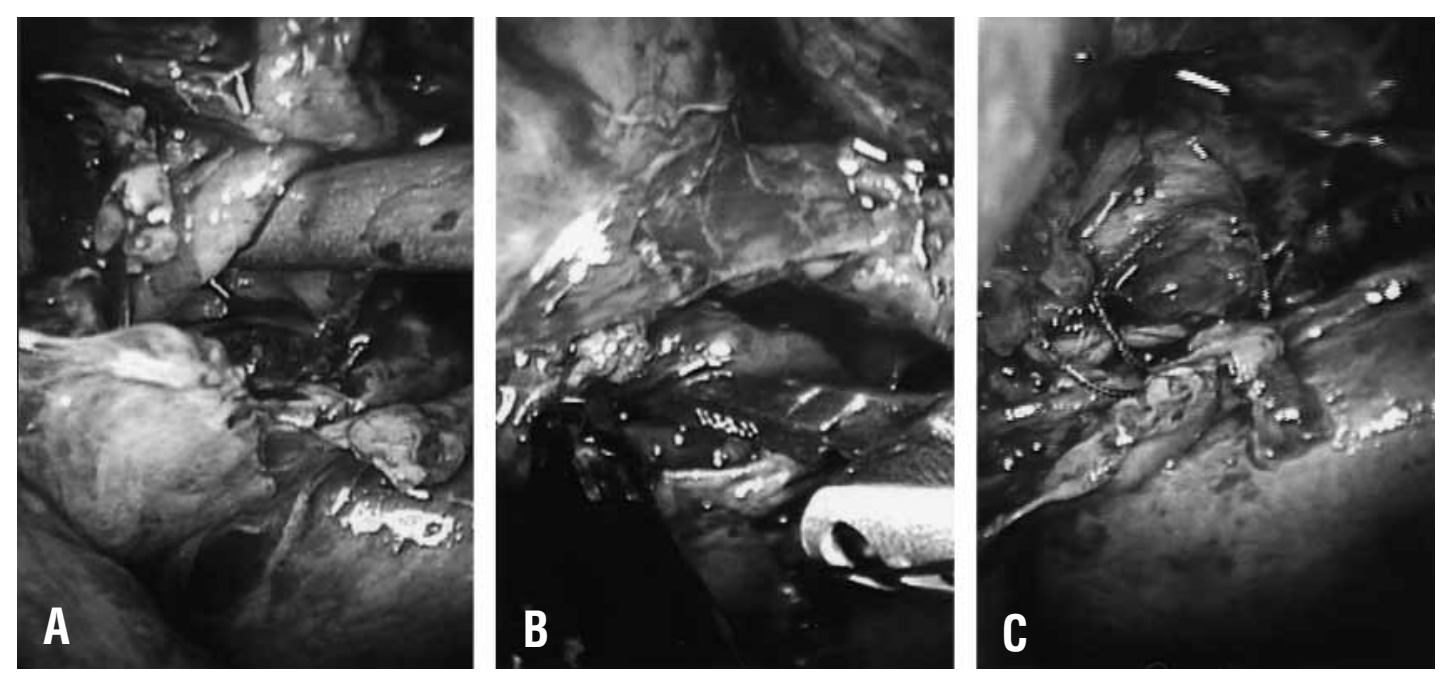

Fig 2. Under thoracoscopic magnification, the longitudinal tear is shown clearly (A), the perforated site of the esophagus is sutured with the Endo Stitch loading unit device (B), and repair is completed (C).

diatrizoate esophagogram 7 days after the operation showed no extravasation and improvement in the shift of the middle third of the esophagus (Fig 1).

Discussion. The general condition of the patient in this disease is usually poor, and excessive trauma should be avoided to minimize postopertative complications. Minimally invasive thoracoscopic technique is particularly well suited for the repair of rupture of the esophagus and wide drainage of the mediastinum. Although more than 300 cases of spontaneous perforation of the esophagus have been reported to our knowledge, only 1 case of thoracoscopic repair has been described. ${ }^{3}$ One of the reasons that surgeons have hesitated to use thoracoscopy to repair a perforated esophagus is that suturing is difficult with a conventional device through thoracoscopy because the suture line and the shaft of forceps are not parallel.

The Endo Stitch loading unit has been used to close the uterus after ureterotomy and to repair inguinal hernias laparoscopically, and it has validated claims of increased safety and ease of use. ${ }^{4}$ In our technique using the Endo Stitch loading unit, we have solved the suture problem and clealy demonstrated two clinical advantages. First, with thoracoscopic magnification we could visualize the proximal and distal edges of the longitudinal tear more clearly than with conventional thoracotomy. When conventional thoracotomy is used, it is usually difficult to keep clear vision as the perforated site is often deep and narrow. Second, the Endo Stitch loading unit allows the surgeon to suture the perforated site easily, quickly, and surely by thoracoscopic maneuvers. As with other types of endoscopic surgery, blood loss is minimized, and this technique lessens the postoperative pain and improves ventilation. Moreover, when conventional thoracotomy is required, thoracoscopic surgery can be converted to the conventional thoracotomy immediately and easily. Therefore, we recommend the use of thoracoscopic surgery with the Endo Stitch loading unit in all patients with spontaneous perforation of the esophagus.

\section{REFERENCES}

1. Sabanathan S, Eng J, Richardson J. Surgical management of intrathoracic oesophageal rupture. Br J Surg 1994;81:863-5.

2. Lawrence DR, Ohri SK, Maxon RE, Townsend ER, Fountain SW. Primary esophageal repair for Boerhaave's syndrome. Ann Thorac Surg 1999;67:818-20.

3. Scott HJ, Rosin RD. Thoracoscopic repair of a transmural rupture of the oesophagus (Boerhaave's syndrome). J R Soc Med 1995;88:414-5.

4. Nagai H, Araki S. Semiautomatic suturing device (Maniceps) for laparoscopic surgery. Surg Endosc 1999;13:191-3. 